\title{
Energy-Efficient Opportunistic Transmission Scheduling for Sparse Sensor Networks with Mobile Relays
}

\author{
Wei Liu, ${ }^{1,2}$ Xiaojie Zhao, ${ }^{3}$ Yuan An, ${ }^{1}$ Xiangjun Dai, ${ }^{1}$ Qingtian Ma, ${ }^{1}$ and Hongjian Guo \\ ${ }^{1}$ State Key Lab of Astronautical Dynamics of China, Xian 710043, China \\ ${ }^{2}$ Beijing University of Posts and Telecommunications, Beijing 100093, China \\ ${ }^{3}$ School of Computer and Information Technology, Beijing Jiaotong University, Beijing 100044, China
}

Correspondence should be addressed to Xiaojie Zhao; 14125221@bjtu.edu.cn

Received 16 February 2016; Revised 1 April 2016; Accepted 11 April 2016

Academic Editor: Anna Pandolfi

Copyright (C) 2016 Wei Liu et al. This is an open access article distributed under the Creative Commons Attribution License, which permits unrestricted use, distribution, and reproduction in any medium, provided the original work is properly cited.

\begin{abstract}
Wireless sensing devices have been widely used in civilian and military applications over the past decade. In some application scenarios, the sensors are sparsely deployed in the field and are costly or infeasible to have stable communication links for delivering the collected data to the destined server. A possible solution is to utilize the motion of entities that are already present in the environment to provide opportunistic relaying services for sensory data. In this paper, we design and propose a new scheduling scheme that opportunistically schedules data transmissions based on the optimal stopping theory, with a view of minimizing the energy consumption on network probes for data delivery. In fact, by exploiting the stochastic characteristics of the relay motion, we can postpone the communication up to an acceptable time deadline until the best relay is found. Simulation results validate the effectiveness of the derived optimal strategy.
\end{abstract}

\section{Introduction}

In the past decade, wireless sensing technology has attracted much attention from researchers and engineers. The traditional communication model from the sensor nodes to the data server is either single-hop or multihop. More recently, some new application scenarios have emerged where realtime and fine-grained sensing is not required [1]. One typical example is animal habitat monitoring, where the goal is to monitor and track the habitats of wild animals in a large nature conservation area $[2,3]$. Another example is the asteroid belt exploration, where the goal is to explore numerous asteroids in the asteroid belt region by picoclass satellite swarms [4]. The aforementioned applications share several unique characteristics. First, it is costly or infeasible to have stable communication links for data delivery. On the one hand, the whole space is very large while the sensor nodes are too sparsely deployed to form a fully connected network. On the other hand, it is very difficult for a sensing device to communicate with the data server directly due to resource constraints on radio communication and energy supply.
Second, the information gathering should be performed without any complex design. For example, it is difficult or even impossible to let the sensing device move to a specific location to facilitate information delivery. Third, the delay of data delivery is usually tolerable by the applications that aim at information gathering from a long-term perspective. As a result, it is an interesting challenge to design an effective and efficient data delivery scheme for these applications.

The key to making this feasible is the ubiquitous existence of mobile relays in many of the target application scenarios. Mobile relays are assumed to be capable of short-range wireless communication and can exchange data with a nearby sensing device or an access point (through which to access the data server) they encounter as a result of their motion [5]. In the case of animal habitat monitoring application, this role can be served by tourists with mobile devices like tablets or smartphones [6]. In the case of asteroid belt exploration, the spacecrafts roving in the space can act as messengers to perform this role [7]. Although this solution has already been used in traditional sensor networks, most of previous researches focus on how to control the motion behaviour of 
the relay to reduce sensor nodes' energy consumption $[8,9]$ or communication delay $[10,11]$. These works cannot be directly used in our target applications, where the sensor nodes are mobile [6] or the motion control is costly [7].

In this paper, we propose an opportunistic transmission scheduling scheme for sparse sensor networks with mobile relays, by means of the optimal stopping approach [12, 13]. In the proposed system, mobile relays that pass by the sensor nodes are capable of carrying and storing the collected data. When mobile relays move into the coverage of access points which are connected to the data server and deployed in or around the monitored region, they can forward the stored data to the access points. This solution has obvious advantages. It does not require performing mobility control operations to mobile relays. In addition, according to the mobile and distributed feature of relays, sensory data can be gathered from a very large and highly distributed scenario. Particularly, by exploiting the stochastic characteristics of the relay motion, we can minimize the energy consumption on network probes for data delivery with the derived optimal rule. The proposed scheme is compared with various heuristic ones to demonstrate its efficacy and efficiency.

The rest of this paper is organized as follows. Section 2 describes the system model. The data delivery scheme based on the optimal stopping theory is proposed and discussed in Section 3. Section 4 evaluated the performance of the proposed solution. Section 5 concludes this paper.

\section{System Model}

We consider a very large region where a number of sensor nodes are sparsely deployed to collect data about objects of interest. Depending on the application requirements, the sensor node can work independently, or a small cluster of sensor nodes can work collaboratively like a "virtual" sensor node [4]. The data report is generated for a period of $\Delta t_{g}$. There are also some access points deployed in or around the monitored region and connected to the data server through the Internet. The mobile relays move freely in the region, and they will occasionally pass by a sensor node or an access point. Their arrivals follow a Poisson process with the parameter $\lambda_{1}$. Let $T_{s m}$ denote the time duration from the instant when a data report is generated to the instant when this data report is picked up by the chosen mobile reply, and $T_{m a}$ denote the time duration it takes the mobile replay to meet the first access point after it carries the data report. It is assumed that the intercontact interval $T_{m a}$ follows an exponential distribution with the parameter $\lambda_{2}$. This assumption is reasonable, since previous researches have proven that the exponential distribution can be treated as the approximate distribution of real opportunistic networks for simplicity $[11,14]$. If a mobile relay arrives at a sensor node $i$, node $i$ will decide whether to choose this mobile relay to deliver the stored data. The data is only forwarded to one mobile relay to guarantee an optimal network throughput [15], and a mobile relay only helps one sensor node for data forwarding so as to reduce its resource consumption [1]. Once the mobile relay contacts with a nearby access point, it forwards the data that it carries to the access point and then to the data server eventually.
During the whole process, the sensor node and the mobile relay have to do network probe actively and periodically for discovering the available next-hop forwarder and sending out the data as soon as possible. The corresponding energy consumption on network probes in unit time can be denoted as $\alpha$ and $\beta$, respectively. Then, the total probe energy is equal to $\alpha T_{s m}+\beta T_{m a}$. On the other hand, it is assumed that the energy for data transmission is the same for a given sender (i.e., sensor node or mobile relay). Besides, the transmission energy may be much smaller than the probe energy due to the small data size or the long probe duration [16-18]. Therefore, for a sensor node $s$, it is important to decide how to choose the most suitable mobile relay $m$ from those passing by, so as to minimize the total energy consumption on network probes. This is the problem we want to solve in this paper.

\section{Data Transmission as an Optimal Stopping Problem}

3.1. Optimal Stopping Theory. This paper studies the problem of finding the optimal time for a sensor node to choose a passing-by mobile relay for data forwarding to minimize the energy consumption on network probes, considering that the relay arrivals reflect the stochastic characteristics of the communication opportunities. Note that a lot of similar problems in many scientific areas, especially in communications, comprise the problem of choosing the best time to take a given action to maximize/minimize an expected payoff/cost [12]. The optimal stopping theory is proposed to solve such problems which chooses the optimal time to make a decision based on sequentially observed random variables [13].

Specifically, stopping rule is defined by two objects: (1) a sequence of random variables, $X_{1}, X_{2}, \ldots$, whose joint distribution is assumed to be known, and (2) a sequence of real-valued reward functions, $y_{0}, y_{1}\left(x_{1}\right), y_{2}\left(x_{1}, x_{2}\right), \ldots$, $y_{\infty}\left(x_{1}, x_{2}, \ldots\right)$. Given these two objects, the associated stopping rule problem can be described as follows. The decision maker observes the sequence of random variables $X_{1}=x_{1}$, $X_{2}=x_{2}, \ldots, X_{n}=x_{n}$. At each step $n$, it can choose to either stop and receive the known reward $y_{n}\left(x_{1}, \ldots, x_{n}\right)$ or continue and observe $X_{n+1}$ for another decision. The goal is to choose a time $0 \leq N \leq \infty$ to stop such that the expected reward $\mathbb{E}\left[Y_{N}=y_{N}\left(x_{1}, \ldots, x_{N}\right)\right]$ is maximized, where $\mathbb{E}[\cdot]$ corresponds to the expected value function. The observation of random variables can be infinite or finite. If there is a known upper bound on the number of stages $N$ at which one has to stop, the problem is called a finite-horizon problem with a horizon $N$. A well-known problem of this type is the "secretary problem" [19]. Such problems can be solved by the method of backward induction from stage $N$ back to the initial stage $0[13,20]$. By now, it has already been used to solve problems on channel exploration in cognitive radios [21], delay minimization in vehicle networks [20], energy saving in WLAN systems [22], and throughput improvement in cooperative networks [23]. The major difference between the proposed scheme and the aforementioned studies is that our scheme exploits the passing-by mobile relays to find the energy-optimal instant to forward the sensory data based on the optimal stopping theory. 
3.2. Energy Oriented Optimal Forwarding. We assume an acceptable time deadline $D_{\max }$ for data forwarding from a sensor node, until which we can postpone the communication. We divide $D_{\max }$ into small time intervals with equal length $\Delta t$, and thus the number of intervals is $N=\left\lfloor D_{\max } / \Delta t\right\rfloor$. Therefore, if the data is forwarded from the sensor in the $n$th interval $(n \in[0, N])$, then $T_{s m} \approx n \Delta t[20]$. In terms of the optimal stopping theory, our problem is to find the stopping time $n$ that minimizes the energy consumption on network probes; that is, $E_{n}\left(T_{m a}\right)=\alpha n \Delta t+\beta T_{m a}$. If the time reaches $D_{\max }$ and the sensor node has not yet forwarded the data, it transmits at $t=D_{\max }$ anyway.

The above objective belongs to the optimal stopping problem with finite horizon, as the sensor node has to stop at no later than stage $N$. In principle, such problem can be solved by the method of backward induction [13]. Since the sensor node must stop at stage $N$, we first find the optimal rule at stage $N-1$. Then, knowing the optimal rule at stage $N-1$, we find the optimal rule at stage $N-2$ and so on back to the first stage. Let $E_{n}$ denote the minimum expected energy to forward data starting from stage $n$; we have

$$
\begin{aligned}
E_{n} & =\min \left\{E_{n}\left(T_{m a}\right), \mathbb{E}\left(E_{n+1}\right)\right\} \\
& =\min \left\{\alpha n \Delta t+\beta T_{m a}, \mathbb{E}\left(E_{n+1}\right)\right\} .
\end{aligned}
$$

We know that, at the last stage $N, \mathbb{E}\left(E_{N}\right)=\mathbb{E}(\alpha N \Delta t+$ $\left.\beta T_{\text {ma }}\right)=\alpha D_{\max }+\beta / \lambda_{2}$. Thus, inductively, we have

$$
\begin{aligned}
& \mathbb{E}\left(E_{n}\right)=\mathbb{E}\left(\min \left\{E_{n}\left(T_{m a}\right), \mathbb{E}\left(E_{n+1}\right)\right\}\right) \\
& =\mathbb{E}\left(\min \left\{\alpha n \Delta t+\beta T_{m a}, \mathbb{E}\left(E_{n+1}\right)\right\}\right) \\
& =\int_{0}^{+\infty} \min \left\{\alpha n \Delta t+\beta T_{m a}, \mathbb{E}\left(E_{n+1}\right)\right\} d F_{T_{m a}}\left(T_{m a}\right) .
\end{aligned}
$$

Because $T_{m a}$ follows the exponential distribution with the parameter $\lambda_{2}$, we have $F_{T_{m a}}(x)=1-e^{-\lambda_{2} x}$ as the cumulative distribution function of $T_{m a}$, and thus $d F_{T_{m a}}(x)=\lambda_{2} e^{-\lambda_{2} x} d x$. Then,

$$
\begin{aligned}
\mathbb{E}\left(E_{n}\right)= & \int_{0}^{E_{n+1}}(\alpha n \Delta t+\beta x) \lambda_{2} e^{-\lambda_{2} x} d x \\
& +\int_{E_{n+1}}^{+\infty} E_{n+1} \lambda_{2} e^{-\lambda_{2} x} d x \\
= & \left(\alpha n \Delta t+\frac{\beta}{\lambda_{2}}\right)\left(1-e^{-\lambda_{2} \mathbb{E}\left(E_{n+1}\right)}\right) \\
& +\mathbb{E}\left(E_{n+1}\right)(1-\beta) e^{-\lambda_{2} \mathbb{E}\left(E_{n+1}\right)} .
\end{aligned}
$$

Thus, we can obtain the optimal rule at each stage $n$; that is, the sensor node is to stop waiting and forward data in the first time interval $k \in[1, N]$ in which $\alpha k \Delta t+\beta T_{m a} \leq \mathbb{E}\left(E_{k+1}\right)$ and to continue otherwise.
3.3. Expected Network Probe Energy. Now, we theoretically analyse the expected energy performance that can be achieved by the new proposed scheme.

Let $\mathbb{E}_{\mathrm{NPE}}$ denote the expected network probe energy, which can be represented as follows:

$$
\begin{aligned}
\mathbb{E}_{\mathrm{NPE}}= & \left(1-P_{\mathrm{NO}, 1}\right) \mathbb{E}_{\mathrm{NPE}, 1} \\
& +\sum_{i=2}^{N}\left(\prod_{l=1}^{i-1} P_{\mathrm{NO}, l}\right)\left(1-P_{\mathrm{NO}, i}\right) \mathbb{E}_{\mathrm{NPE}, i},
\end{aligned}
$$

where $P_{\mathrm{NO}, l}$ denotes the probability that there is no relay chosen to carry data in the $l$ th time interval and $\mathbb{E}_{\mathrm{NPE}, i}$ denotes the expected network probe energy that is carried by a mobile relay in the $i$ th time interval. Since mobile relays arrive according to a Poisson process, we can calculate $P_{\mathrm{NO}, l}$ as follows:

$$
P_{\mathrm{NO}, l}=\sum_{j=0}^{\infty} P_{n}(j)\left(P_{\mathrm{NO} m, l}\right)^{j} .
$$

$P_{n}(j)$ denotes the probability that $j$ mobile relays arrive within $\Delta t$ and $P_{\mathrm{NO}, l}$ represents the probability that a mobile relay arrives within the $l$ th interval but is not qualified to carry the sensory data due to $\alpha l \Delta t+\beta T_{m a}>\mathbb{E}\left(E_{l+1}\right)$. Since the relay arrival follows a Poisson distribution, then it is easy to know that

$$
P_{n}(j)=e^{-\lambda_{1} \Delta t} * \frac{\left(\lambda_{1} \Delta t\right)^{j}}{j !} .
$$

Besides,

$$
\begin{aligned}
P_{\mathrm{NO}, l} & =\operatorname{Pr}\left(\alpha l \Delta t+\beta T_{m a}>\mathbb{E}\left(E_{l+1}\right)\right) \\
& =1-\operatorname{Pr}\left(\alpha l \Delta t+\beta T_{m a} \leq \mathbb{E}\left(E_{l+1}\right)\right) \\
& =1-F_{T_{m a}}\left(\frac{\mathbb{E}\left(E_{l+1}\right)-\alpha l \Delta t}{\beta}\right) \\
& =e^{-\left(\lambda_{2} / \beta\right)\left(\mathbb{E}\left(E_{l+1}\right)-\alpha l \Delta t\right)} .
\end{aligned}
$$

According to the stopping rule, we can obtain the value of $\mathbb{E}_{\mathrm{NPE}, i}$ as follows:

$$
\begin{aligned}
& \mathbb{E}_{\mathrm{NPE}, i}=\beta \mathbb{E}\left(T_{m a} \mid T_{m a} \leq \frac{\mathbb{E}\left(E_{i+1}\right)-\alpha i \Delta t}{\beta}\right)+\alpha i \Delta t \\
& =\beta \frac{\int_{0}^{\left(\mathbb{E}\left(E_{i+1}\right)-\alpha i \Delta t\right) / \beta} x d F_{T_{m a}(x)}}{F_{T_{m a}\left(\mathbb{E}\left(E_{i+1}\right)-\alpha i \Delta t\right)}}+\alpha i \Delta t \\
& =\beta \frac{1 / \lambda_{2}-\left(\left(\mathbb{E}\left(E_{i+1}\right)-\alpha i \Delta t\right) / \beta+1 / \lambda_{2}\right) e^{-\left(\lambda_{2} / \beta\right)\left(\mathbb{E}\left(E_{i+1}\right)-\alpha i \Delta t\right)}}{1-e^{-\left(\lambda_{2} / \beta\right)\left(\mathbb{E}\left(E_{i+1}\right)-\alpha i \Delta t\right)}}
\end{aligned}
$$

$+\alpha i \Delta t$

Finally, we can obtain the expected network probe energy by $(4)-(8)$. 


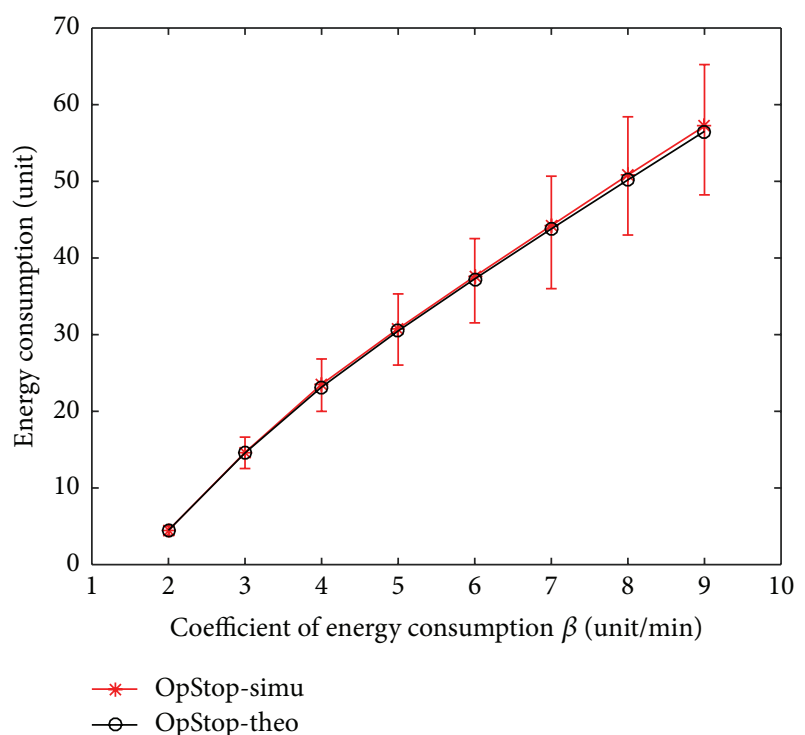

FIGURE 1: Network probe energy consumption with regard to $\beta$.

\section{Performance Evaluation}

In this section, we evaluate the performance of the proposed models and solution. Specifically, we present the simulation model, the valuation metrics, the numerical results of the proposed scheme for different scenarios, and the simulation benchmarking results by comparing the proposed scheme with some heuristics. In our Matlab-based simulation, the sensor nodes are sparsely and randomly deployed in a large area and generate data every $\Delta t_{g}=5$ minutes. Besides, we set $\Delta t=0.5$ minutes. To make the results more pronounced, for each specific scenario, the experiment is carried out independently for 500 rounds. Note that we do not give a specific energy unit here because different sensor nodes have distinct energy models $[7,8]$ but simply use "unit" instead.

In the first set of simulations, we fix $\lambda_{1}=4, \lambda_{2}=1 / 15$, and $\alpha=0.2$ and then run simulation experiments with different $\beta$ values. In Figure 1, the impact of the relationship between probe power parameters, that is, $\alpha$ and $\beta$, is shown. We can notice that, with the increase of $\beta$, the total energy consumption on network probes increases. Besides, the result fluctuations will become larger when the ratio $\beta / \alpha$ grows.

In the second set of simulations, we fix $\alpha=0.2, \beta=$ 1 , and $\lambda_{2}=1 / 15$ and then run simulation experiments with different $\lambda_{1}$ values. In Figure 2, the impact of the arrival rate of mobile relay, that is, $\lambda_{1}$, is shown. With the increase of $\lambda_{1}$, there will be more mobile relays passing by the sensor node in the former intervals. This will increase the forwarding probability in these intervals, resulting in lower energy consumption on network probes.

In the third set of simulations, we fix $\alpha=0.2, \beta=$ 1 , and $\lambda_{1}=4$ and then run simulation experiments with different $\lambda_{2}$ values. In Figure 3, the impact of the mean time in which a mobile relay meets an access point, that is, $1 / \lambda_{2}$, is shown. A larger value of $\lambda_{2}$ implies that the value of $T_{m a}$ is

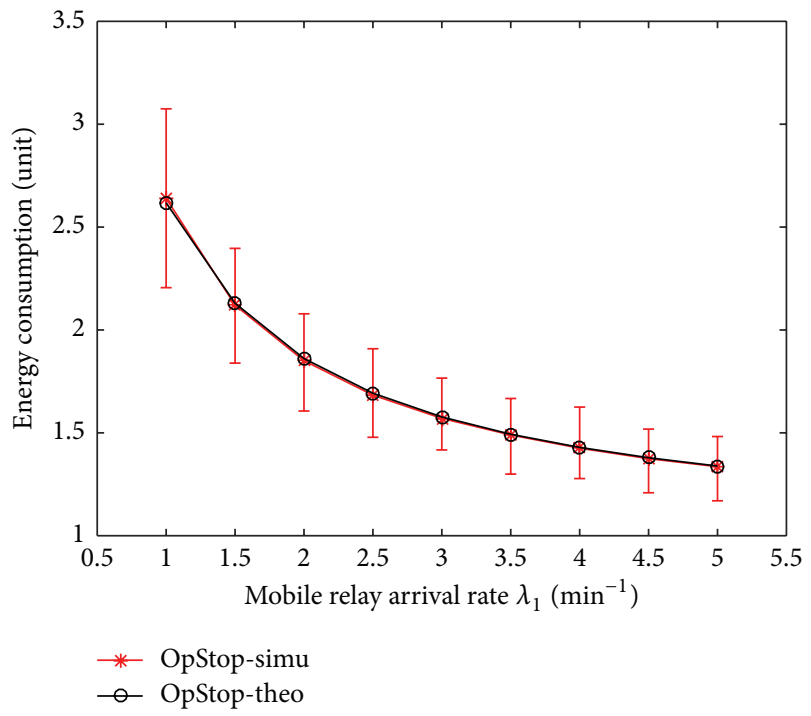

FIGURE 2: Network probe energy consumption with regard to $\lambda_{1}$.

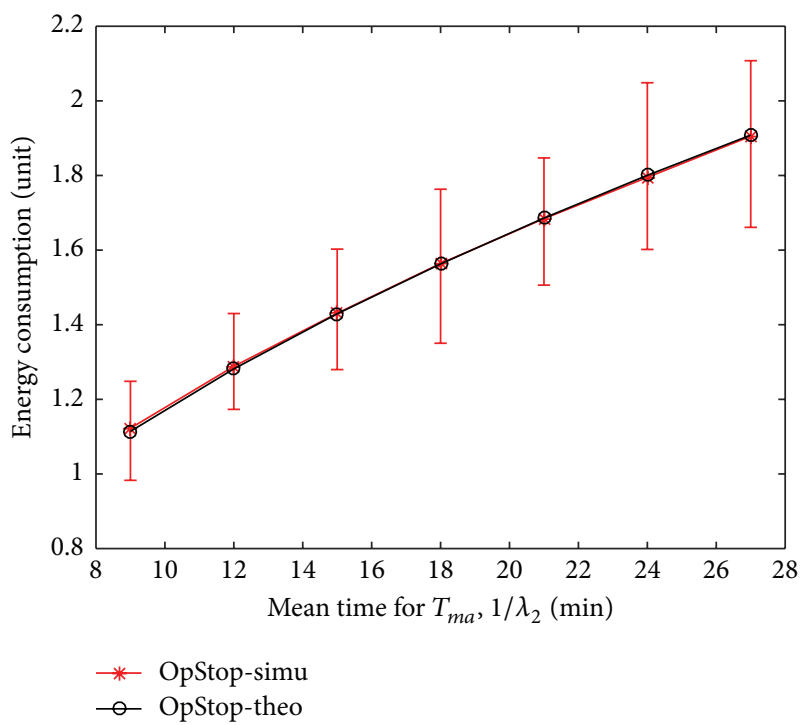

FIGURE 3: Network probe energy consumption with regard to $\lambda_{2}$.

smaller, resulting in lower energy consumption on network probes.

In the fourth set of simulations, we fix $\alpha=0.2, \lambda_{1}=$ 4 , and $\lambda_{2}=1 / 15$ and then run simulation experiments with different $D_{\max }$ values. In Figure 4, we can notice that $D_{\max }$ has little impact on the energy consumption with the same settings of $\alpha$ and $\beta$. The results indicate the performance stability of the proposed scheme. However, if the value of $D_{\max }$ and $N$ is very small (e.g., $D_{\max }=5$ and $N=$ 10 ), it may be difficult to find the most optimal forwarder in some cases, leading to some additional energy cost.

We then compare the proposed scheme with three heuristic schemes. These heuristic schemes are based on either simple statistics (representing some long-term statistical 


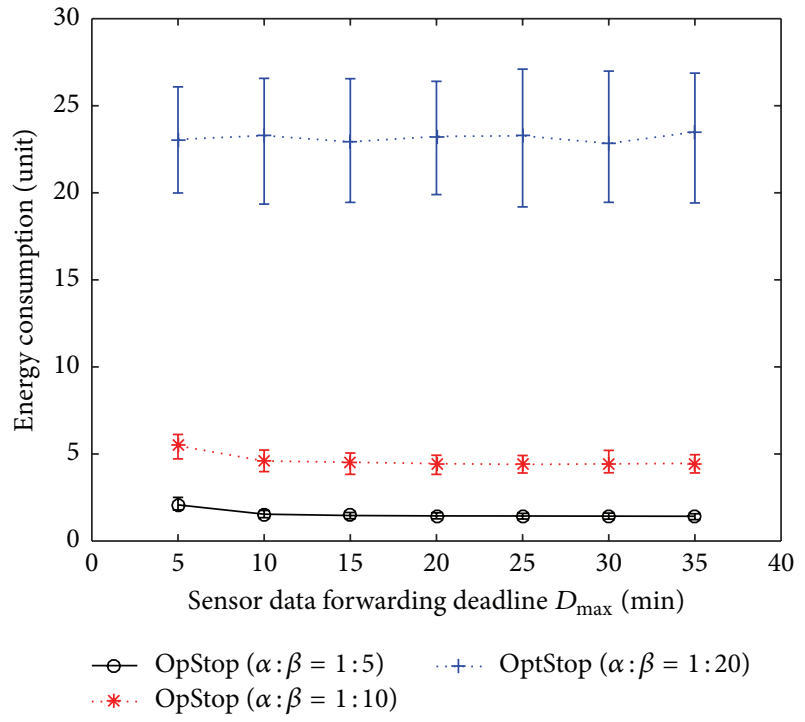

FIGURE 4: Network probe energy consumption with regard to $D_{\max }$.

methods) or simple solutions from other optimal stopping problems [20]:

(1) Deterministic forwarding scheme (DFS): this is a simple method that schedules the forwarding at constant equidistant time instants, $D_{\max }$. This heuristic, despite its simplicity, is necessary for comparison.

(2) Random forwarding scheme (RFS): the RFS method does not prompt the sensor node to transmit at specific time instants but at a random time within the time length $\left[0, D_{\max }\right]$.

(3) Average-energy forwarding scheme (AFS): the AFS method is based on the average observed energy computed from the beginning to the current interval. The decision on whether to forward or postpone is based on the comparison of the current energy value with the energy average of the previous intervals. If the former is smaller than the latter, then the data is forwarded immediately. Otherwise, the data forwarding will be postponed.

We carried out a series of simulations for comparison, using the same parameter settings as those in the first three set of experiments, respectively (except that in Figure 8 we set $\alpha=0.2$ and $\beta=1$ ). From the results shown in Figures $5-8$, it is obvious that the proposed scheme outperforms the others in terms of energy efficiency, especially when the value of parameters (i.e., $\beta, \lambda_{1}$, and $\lambda_{2}$ ) is relatively large. Besides, the result fluctuations of the proposed scheme is the smallest among the four schemes. The RFS and DFS schemes do not take any energy factor into consideration, so they have the worst performance. The AFS scheme only considers the energy consumption in the past intervals and thus may stop prematurely at a local minimum and cannot make the optimal decision.

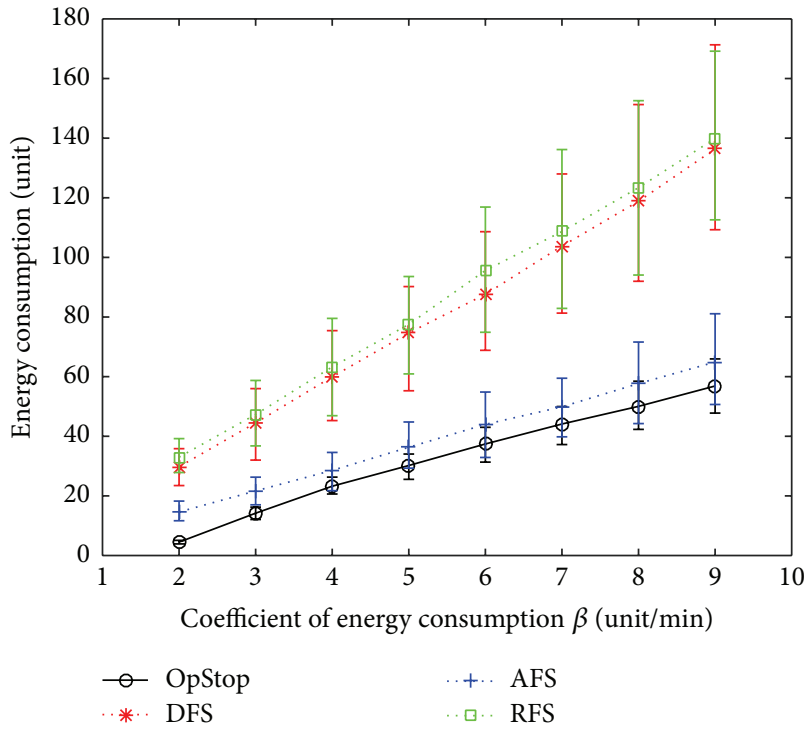

FIGURE 5: Network probe energy consumption with regard to $\beta$ under different schemes.

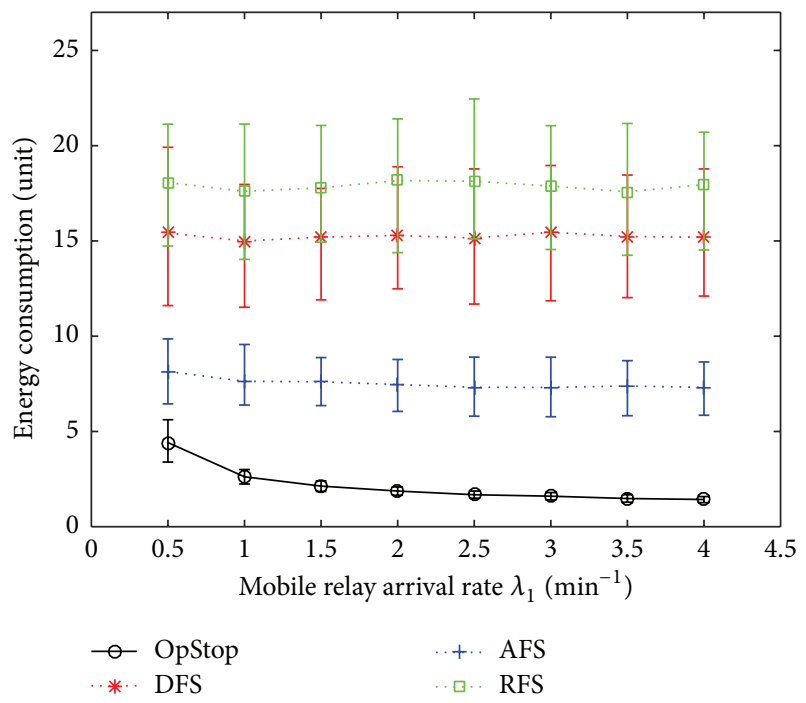

FIGURE 6: Network probe energy consumption with regard to $\lambda_{1}$ under different schemes.

\section{Conclusion}

One of the key concerns in wireless sensor networks is the design of energy-efficient transmission policies. This paper presented a new scheduling scheme that minimizes the energy consumption on network probes in sparse sensor networks, where it is difficult to set up stable communication links for gathering sensory data. Based on the optimal stopping theory, which is about how to find the optimal time instant to make an optimal decision, we exploited mobile relays to design an opportunistic transmission scheduling 


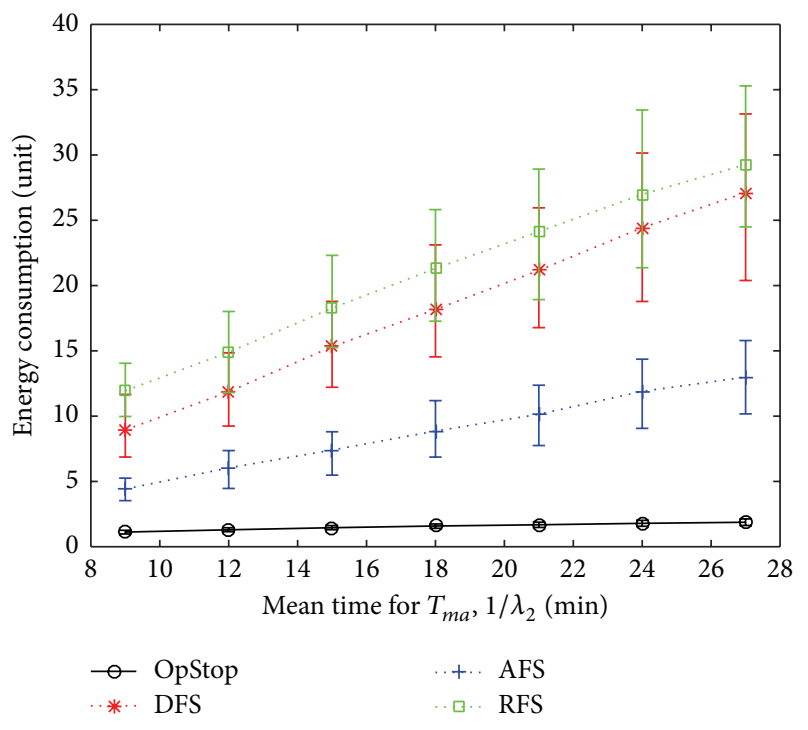

FIGURE 7: Network probe energy consumption with regard to $\lambda_{2}$ under different schemes.

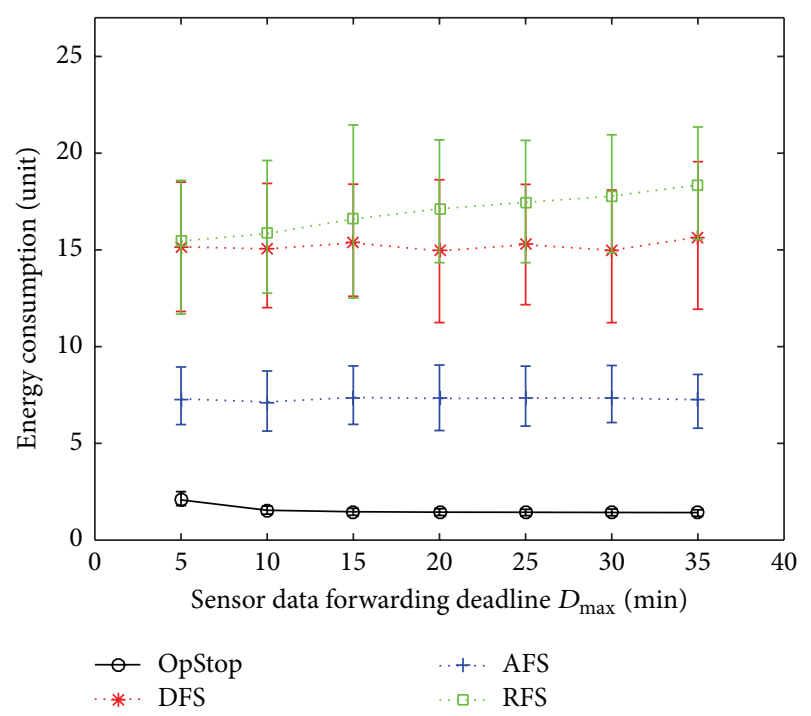

FIGURE 8: Network probe energy consumption with regard to $D_{\max }$ under different schemes.

scheme. More specifically, the sensor node postpones forwarding data until it finds the most suitable passing-by relay, which would minimize the energy on network probes for data delivery under specific time deadline constraints. The simulation results have shown that the energy performance of our scheme is quite promising and energy saving is significant as compared to various heuristic schemes. In the future, we plan to conduct an experimental campaign in real test-beds to validate our scheme.

\section{Competing Interests}

The authors declare that they have no competing interests.

\section{Acknowledgments}

This work was supported in part by the State Key Lab of Astronautic Dynamics of China under Grant no. 2015ADLDW0401 and the National Science Foundation of China (NSFC) under Grant no. 61401516.

\section{References}

[1] G. Anastasi, M. Conti, and M. Di Francesco, "An analytical study of reliable and energy-efficient data collection in sparse sensor networks with mobile relays," in Wireless Sensor Networks: 6th European Conference, EWSN 2009, Cork, Ireland, February 11-13, 2009. Proceedings, U. Roedig and C. Sreenan, Eds., vol. 5432 of Lecture Notes in Computer Science, pp. 199215, Springer, Berlin, Germany, 2009.

[2] R. R. Fletcher, K.-I. Amemori, M. Goodwin, and A. M. Graybiel, "Wearable wireless sensor platform for studying autonomic activity and social behavior in non-human primates," in Proceedings of the 34th Annual International Conference of the IEEE Engineering in Medicine and Biology Society (EMBS '12), pp. 4046-4049, San Diego, Calif, USA, August 2012.

[3] F. H. Bijarbooneh, P. Flener, E. Ngai, and J. Pearson, "Optimising quality of information in data collection for mobile sensor networks," in Proceedings of the IEEE/ACM 21st International Symposium on Quality of Service (IWQoS '13), pp. 1-10, IEEE, Montreal, Canada, June 2013.

[4] T. Vladimirova, X. Wu, and C. P. Bridges, "Development of a satellite sensor network for future space missions," in Proceedings of the IEEE Aerospace Conference (AC '08), Big Sky, Mont, USA, March 2008.

[5] R. C. Shah, S. Roy, S. Jain, and W. Brunette, "Data MULEs: modeling and analysis of a three-tier architecture for sparse sensor networks," Ad Hoc Networks, vol. 1, no. 2-3, pp. 215-233, 2003.

[6] J. Zhang, C. Chen, J. Ma, N. He, and Y. Ren, "USink: smartphone-based moible sink for wireless sensor networks," in Proceedings of the IEEE Consumer Communications and Networking Conference (CCNC '11), pp. 90-95, IEEE, Las Vegas, Nev, USA, January 2011.

[7] E. Vassev, R. Sterritt, C. Rouff, and M. Hinchey, "Swarm technology at NASA: building resilient systems," IT Professional, vol. 14, no. 2, pp. 36-42, 2012.

[8] J. Luo and J.-P. Hubaux, "Joint mobility and routing for lifetime elongation in wireless sensor networks," in Proceedings of the 24th Annual Joint Conference of the IEEE Computer and Communications Societies (IEEE INFOCOM '05), vol. 3, pp. 1735-1746, Miami, Fla, USA, March 2005.

[9] W. Wang, V. Srinivasan, and K.-C. Chua, "Extending the lifetime of wireless sensor networks through mobile relays," IEEE/ ACM Transactions on Networking, vol. 16, no. 5, pp. 1108-1120, 2008.

[10] R. Sugihara and R. Gupta, "Improving the data delivery latency in sensor networks with controlled mobility," in Distributed Computing in Sensor Systems: 4th IEEE International Conference, DCOSS 2008 Santorini Island, Greece, June 11-14, 2008 Proceedings, S. Nikoletseas, B. Chlebus, D. Johnson, and B. Krishnamachari, Eds., vol. 5067, pp. 386-399, Springer, Berlin, Germany, 2008.

[11] M. Zhao and Y. Yang, "Bounded relay hop mobile data gathering in wireless sensor networks," IEEE Transactions on Computers, vol. 61, no. 2, pp. 265-277, 2012. 
[12] H. Robbins, Y. S. Chow, and D. Siegmund, Great Expectations: The Theory of Optimal Stopping, Houghton Mifflin, New York, NY, USA, 1971.

[13] T. S. Ferguson, Optimal Stopping and Applications, 2006, http:// www.math.ucla.edu/ tom/Stopping/Contents.html.

[14] Y. Li, D. Jin, L. Zeng, and S. Chen, "Revealing patterns of opportunistic contact durations and intervals for large scale urban vehicular mobility," in Proceedings of the IEEE International Conference on Communications (ICC '13), pp. 1646-1650, IEEE, Budapest, Hungary, June 2013.

[15] N. Lu, T. H. Luan, M. Wang, X. Shen, and F. Bai, "Capacity and delay analysis for social-proximity urban vehicular networks," in Proceedings of the IEEE INFOCOM, pp. 1476-1484, IEEE, Orlando, Fla, USA, March 2012.

[16] W. Wang, M. Motani, and V. Srinivasan, "Opportunistic energy-efficient contact probing in delay-tolerant applications," IEEE/ACM Transactions on Networking, vol. 17, no. 5, pp. 15921605, 2009.

[17] X. Wu, K. N. Brown, and C. J. Sreenan, "Data pre-forwarding for opportunistic data collection in wireless sensor networks," ACM Transactions on Sensor Networks, vol. 11, no. 1, article 8, 33 pages, 2014.

[18] X. Wu, K. N. Brown, and C. J. Sreenan, "Contact probing mechanisms for opportunistic sensor data collection," The Computer Journal, vol. 58, no. 8, pp. 1792-1810, 2015.

[19] P. R. Freeman, "The secretary problem and its extensions: a review," International Statistical Review, vol. 51, no. 2, pp. 189206, 1983.

[20] N. Cheng, N. Lu, N. Zhang, X. S. Shen, and J. W. Mark, "Vehicleassisted data delivery for smart grid: an optimal stopping approach," in Proceedings of the IEEE International Conference on Communications (ICC '13), pp. 6184-6188, Budapest, Hungary, June 2013.

[21] Y. Xu, J. Wang, Q. Wu, Z. Zhang, A. Anpalagan, and L. Shen, "Optimal energy-efficient channel exploration for opportunistic spectrum usage," IEEE Wireless Communications Letters, vol. 1, no. 2, pp. 77-80, 2012.

[22] S. Chen, X. Gan, and H. Li, "Energy efficiency driven random access in WLAN system," in Proceedings of the International Conference on Wireless Communications \& Signal Processing (WCSP '12), pp. 1-6, IEEE, Huangshan, China, October 2012.

[23] X. Gong, C. T. Ps, J. Zhang, and H. V. Poor, "Opportunistic cooperative networking: to relay or not to relay?" IEEE Journal on Selected Areas in Communications, vol. 30, no. 2, pp. 307-314, 2012. 


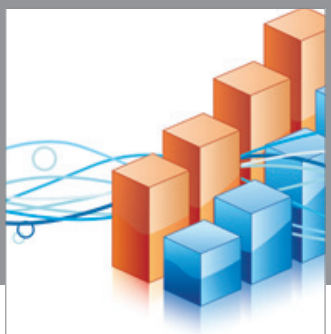

Advances in

Operations Research

vatem alat4

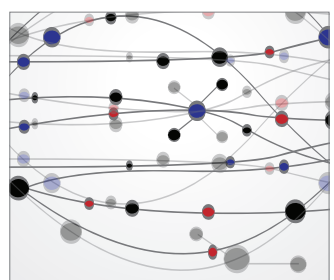

\section{The Scientific} World Journal
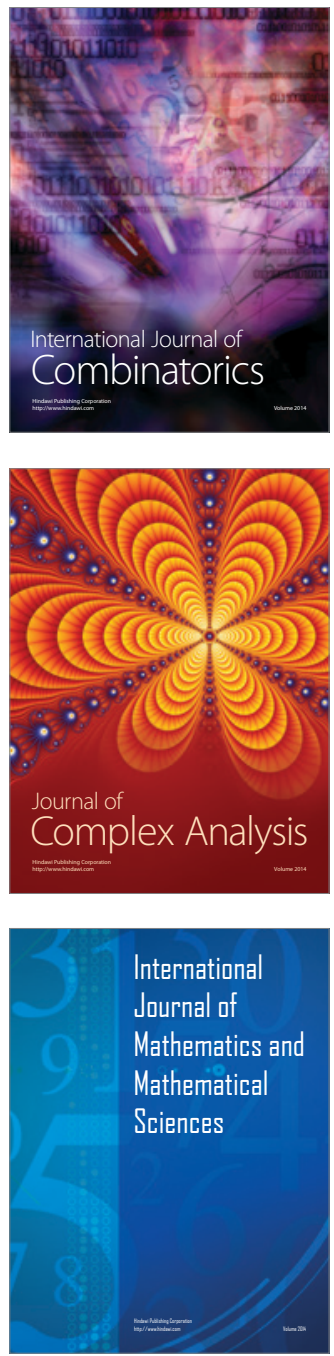
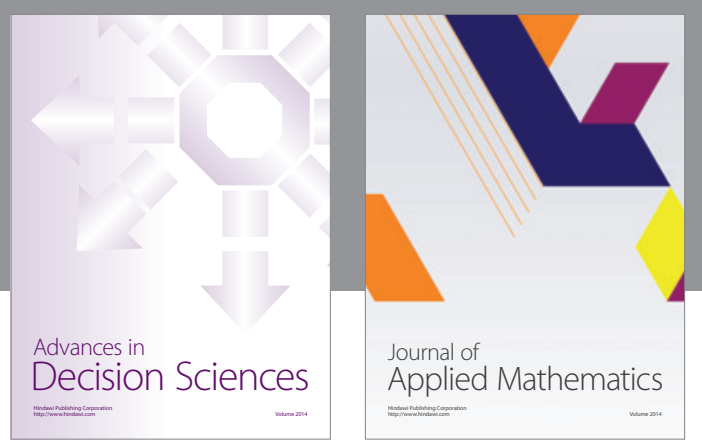

Algebra

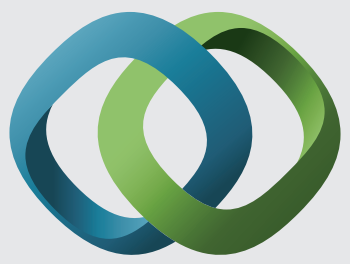

\section{Hindawi}

Submit your manuscripts at

http://www.hindawi.com
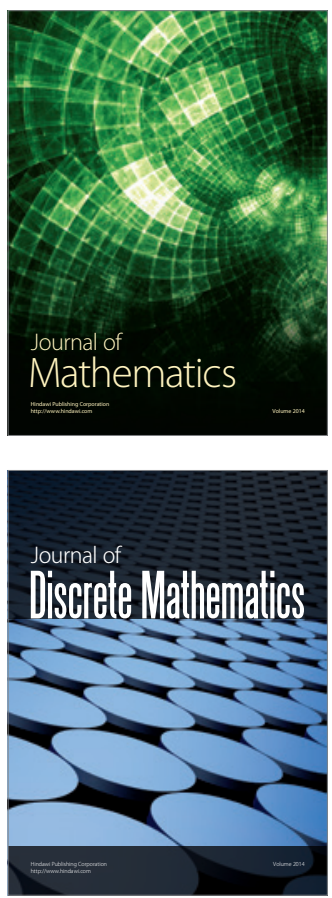

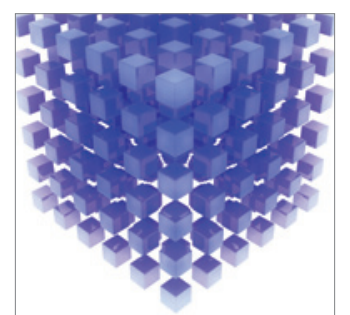

Mathematical Problems in Engineering
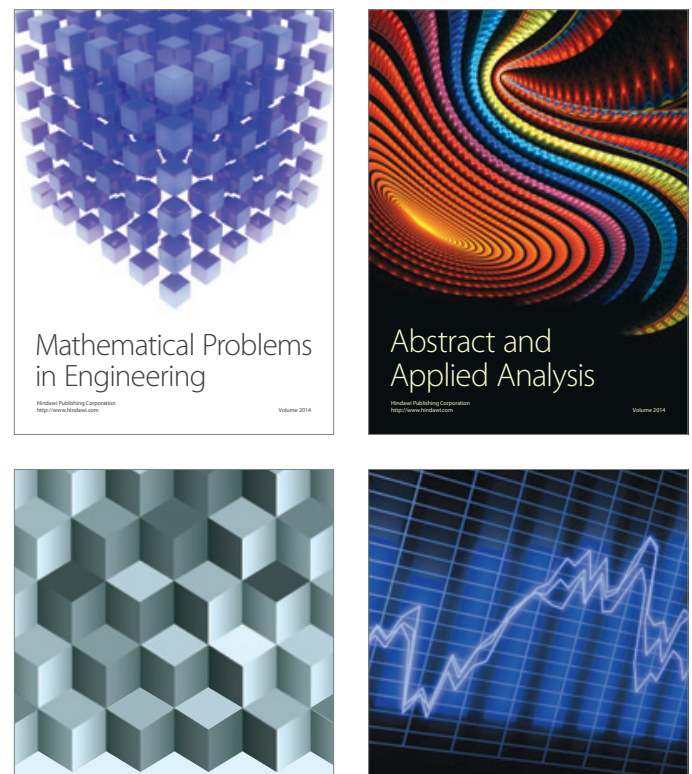

Journal of

Function Spaces

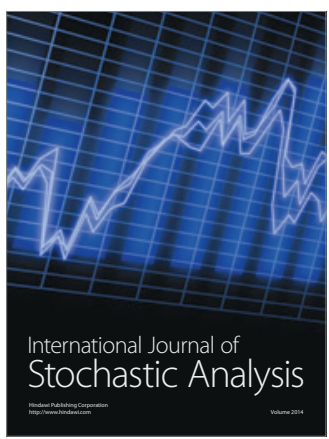

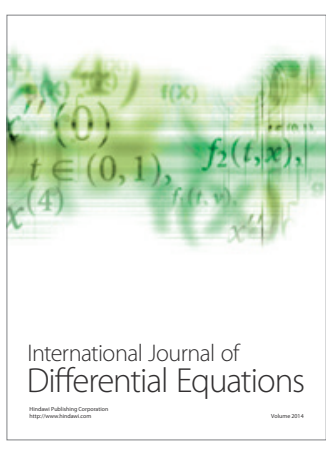
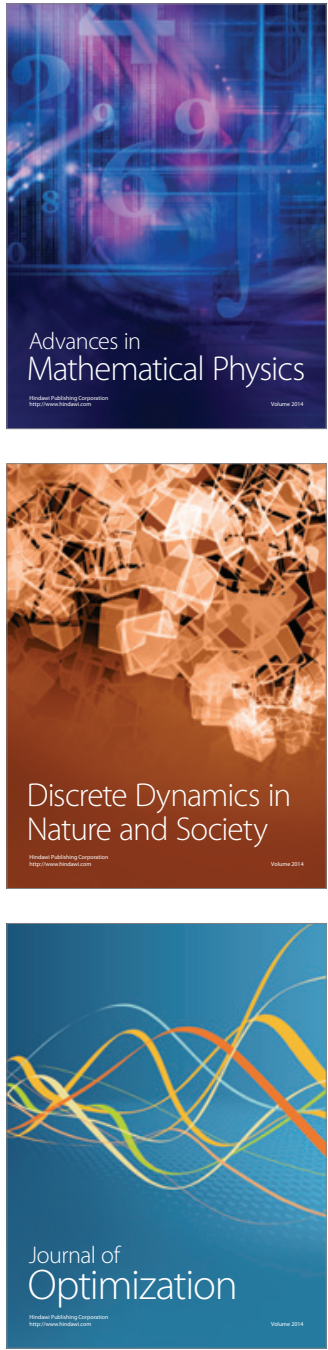